\title{
Effective Hamiltonian Approach to Hyperon Beta Decay with Final-State Baryon Polarization
}

\author{
S. Bright and R. Winston \\ The Enrico Fermi Institute and the Department of Physics, \\ The University of Chicago, Chicago, Illinois 60637 \\ E. C. Swallow \\ Department of Physics, Elmhurst College, Elmhurst, Illinois 60126 and \\ The Enrico Fermi Institute, The University of Chicago, Chicago, Illinois 60637 \\ A. Alavi-Harati \\ University of Wisconsin, Madison, Wisconsin, 53706
}

(November 30, 2017)

\begin{abstract}
Using an effective Hamiltonian approach, we obtain expressions for hyperon $\beta$ decay final-state baryon polarization. Terms through second order in the energy release are retained. The resulting approximate expressions are much simpler and more compact than the exact expressions, and they agree closely with them.
\end{abstract}

PACS numbers: 13.30.Ce

Typeset using REVTEX 
In decays such as $\Xi^{-} \rightarrow \Lambda e^{-} \bar{\nu}$ or the recently observed $[1] \Xi^{0} \rightarrow \Sigma^{+} e^{-} \bar{\nu}$, the decay form factors can be probed by observing the parity-violating polarization of the final-state baryon via its two body decay mode $\left(\Lambda \rightarrow p \pi^{-}\right.$or $\Sigma^{+} \rightarrow p \pi^{0}$ respectively). In addition, other kinematic distributions can be evaluated in the rest frame of the final baryon.

Early analyses of hyperon $\beta$ decay with the final-state polarization observed were restricted to the zero-recoil approximation [2] or were limited in scope [3]. More recent detailed treatments exist [4, [5], but the resulting expressions are quite opaque, and, as a result, the physical content is hidden. Also, experiments are not likely to require the exact formulae within the foreseeable future.

Using a method introduced by Primakoff for muon capture [6,7], we keep only terms through second order in the recoil velocity of the initial baryon (in the rest frame of the final baryon). A similar approach has been used to derive expressions for the case of a polarized initial baryon [8].

The most general $\mathrm{V}$-A transition matrix element for the generic hyperon $\beta$ decay process $B \rightarrow b e^{-} \bar{\nu}$ can be written [9] in the form

$$
\mathcal{M}=G_{S} \frac{\sqrt{2}}{2} \bar{u}_{b}\left(O_{\alpha}^{V}+O_{\alpha}^{A}\right) u_{B} \bar{u}_{e} \gamma^{\alpha}\left(1+\gamma_{5}\right) v_{\nu}+H . c .
$$

where

$$
\begin{gathered}
O_{\alpha}^{V}=f_{1} \gamma_{\alpha}+\frac{f_{2}}{M_{B}} \sigma_{\alpha \beta} q^{\beta}+\frac{f_{3}}{M_{B}} q_{\alpha}, \\
O_{\alpha}^{A}=\left(g_{1} \gamma_{\alpha}+\frac{g_{2}}{M_{B}} \sigma_{\alpha \beta} q^{\beta}+\frac{g_{3}}{M_{B}} q_{\alpha}\right) \gamma_{5}, \\
q^{\alpha}=\left(p_{e}+p_{\nu}\right)^{\alpha}=\left(p_{B}-p_{b}\right)^{\alpha}, \text { and } \\
G_{S}=\left\{\begin{array}{l}
G_{F} V_{u s} \text { for }|\Delta S|=1 \\
G_{F} V_{u d} \text { for } \Delta S=0 .
\end{array}\right.
\end{gathered}
$$

Here $G_{F}$ is the Fermi coupling constant, $V_{u s}$ and $V_{u d}$ are the appropriate CabibboKobayashi-Maskawa matrix elements, and $\Delta S$ denotes the strangeness change in the decay. We relate the transition matrix element to an effective Hamiltonian by 


$$
\mathcal{M}=\left\langle b e\left|\mathcal{H}_{\text {eff }}\right| B \nu\right\rangle \sqrt{2 e 2 \nu 2 M_{b}\left(E_{B}+M_{B}\right)}
$$

with

$$
\begin{aligned}
\frac{\sqrt{2}}{2} \mathcal{H}_{\mathrm{eff}}= & G_{S} \frac{1}{2}\left(1-\sigma_{\ell} \cdot \hat{e}\right)\left[G_{V}+G_{A} \sigma_{\ell} \cdot \sigma_{\mathbf{b}}\right. \\
& \left.+G_{P}^{e} \sigma_{\mathbf{b}} \cdot \hat{e}+G_{P}^{\nu} \sigma_{\mathbf{b}} \cdot \hat{\nu}\right] \frac{1}{2}\left(1-\sigma_{\ell} \cdot \hat{\nu}\right) .
\end{aligned}
$$

Here $\hat{e}$ and $\hat{\nu}$ are unit vectors along the electron and antineutrino directions, while $e, \nu$, and $E_{B}$ are the energies of the electron, antineutrino, and initial baryon (all quantities are in the rest frame of $b$ ). The spin operators $\sigma_{\ell}$ and $\sigma_{\mathbf{b}}$ act respectively on the lepton and baryon states (represented by two-component spinors).

The effective coupling coefficients $G_{V}, G_{A}, G_{P}^{e}$, and $G_{P}^{\nu}$ are functions of the form factors in eqn. (2):

$$
\begin{aligned}
& G_{V}=f_{1}+\delta f_{2}-\frac{\nu+e}{2 M_{B}}\left(f_{1}+\Delta f_{2}\right), \\
& G_{A}=-g_{1}+\delta g_{2}+\frac{\nu-e}{2 M_{B}}\left(f_{1}+\Delta f_{2}\right), \\
& G_{P}^{e}=\frac{e}{2 M_{B}}\left(-\left(f_{1}+\Delta f_{2}\right)-g_{1}+\Delta g_{2}\right), \\
& G_{P}^{\nu}=\frac{\nu}{2 M_{B}}\left(f_{1}+\Delta f_{2}-g_{1}+\Delta g_{2}\right),
\end{aligned}
$$

where $\delta=\left(M_{B}-M_{b}\right) / M_{B}$ and $\Delta=\left(M_{B}+M_{b}\right) / M_{B}=2-\delta$. Since the form factors $f_{3}$ and $g_{3}$ always appear with a multiplier of the electron mass divided by $M_{B}$, they are neglected throughout. Note also that $f_{2}$ and $g_{2}$ always appear multiplied by a quantity of order $\delta$, so their $q^{2}$ dependence is not relevant to our order $\delta^{2}$ approximation. However, the $q^{2}$ dependence of $f_{1}$ and $g_{1}$ does need to be included [5] in calculations to maintain a completely consistent order of approximation.

Electron and antineutrino spins are not usually observed, and this analysis focuses on measurement of the final baryon polarization. We therefore sum over the electron and antineutrino spins and average over initial baryon spin:

$$
\sum_{\nu \text { spins }, B \text { spins }}\left|\left\langle b e\left|\mathcal{H}_{\text {eff }}\right| B \nu\right\rangle\right|^{2}=\left\langle b e\left|\mathcal{H}_{\text {eff }} \mathcal{H}_{\text {eff }}^{\dagger}\right| b e\right\rangle
$$


and

$$
\sum_{e \text { spins }}\left\langle b e\left|\mathcal{H}_{\text {eff }} \mathcal{H}_{\text {eff }}^{\dagger}\right| b e\right\rangle=\operatorname{Tr}\left(\left(1+\sigma_{\mathbf{b}} \cdot \mathbf{P}_{\mathbf{b}}\right) \mathcal{H}_{\text {eff }} \mathcal{H}_{\text {eff }}^{\dagger}\right)
$$

By projecting out the spin of the final baryon and taking the trace, we obtain

$$
\begin{aligned}
& |\mathcal{M}|^{2}=\xi\left[1+a \hat{e} \cdot \hat{\nu}+A \mathbf{P}_{\mathbf{b}} \cdot \hat{e}+B \mathbf{P}_{\mathbf{b}} \cdot \hat{\nu}\right. \\
& +A^{\prime}\left(\mathbf{P}_{\mathbf{b}} \cdot \hat{e}\right)(\hat{e} \cdot \hat{\nu})+B^{\prime}\left(\mathbf{P}_{\mathbf{b}} \cdot \hat{\nu}\right)(\hat{e} \cdot \hat{\nu}) \\
& \left.+D \mathbf{P}_{\mathbf{b}} \cdot(\hat{e} \times \hat{\nu})\right] \\
& \cdot(2 e)(2 \nu)\left(2 M_{b}\right)\left(E_{B}+M_{B}\right) G_{S}^{2}, \\
& \xi=\left|G_{V}\right|^{2}+3\left|G_{A}\right|^{2}-2 \operatorname{Re}\left(G_{A}^{*}\left(G_{P}^{e}+G_{P}^{\nu}\right)\right) \\
& +\left|G_{P}^{e}\right|^{2}+\left|G_{P}^{\nu}\right|^{2} \\
& \xi a=\left|G_{V}\right|^{2}-\left|G_{A}\right|^{2}-2 \operatorname{Re}\left(G_{A}^{*}\left(G_{P}^{e}+G_{P}^{\nu}\right)\right) \\
& +\left|G_{P}^{e}\right|^{2}+\left|G_{P}^{\nu}\right|^{2}+2 \operatorname{Re}\left(G_{P}^{e *} G_{P}^{\nu}\right)(1+\hat{e} \cdot \hat{\nu}), \\
& \xi A=-2 \operatorname{Re}\left(G_{V}^{*} G_{A}\right)+2\left|G_{A}\right|^{2} \\
& +2 \operatorname{Re}\left(G_{V}^{*} G_{P}^{e}-G_{A}^{*} G_{P}^{\nu}\right), \\
& \xi B=-2 \operatorname{Re}\left(G_{V}^{*} G_{A}\right)-2\left|G_{A}\right|^{2} \\
& +2 \operatorname{Re}\left(G_{V}^{*} G_{P}^{\nu}+G_{A}^{*} G_{P}^{e}\right), \\
& \xi A^{\prime}=2 \operatorname{Re}\left(G_{P}^{e *}\left(G_{V}-G_{A}\right)\right), \\
& \xi B^{\prime}=2 \operatorname{Re}\left(G_{P}^{\nu *}\left(G_{V}+G_{A}\right)\right), \\
& \xi D=2 \operatorname{Im}\left(G_{V}^{*} G_{A}\right)+2 \operatorname{Im}\left(G_{P}^{e *} G_{P}^{\nu}\right)(1+\hat{e} \cdot \hat{\nu}) \\
& +2 \operatorname{Im}\left(G_{A}^{*}\left(G_{P}^{e}-G_{P}^{\nu}\right)\right) .
\end{aligned}
$$

The polarization of the final baryon may be expressed explicitly as

$$
\mathbf{P}_{\mathbf{b}}=\frac{\left(A+A^{\prime} \hat{e} \cdot \hat{\nu}\right) \hat{e}+\left(B+B^{\prime} \hat{e} \cdot \hat{\nu}\right) \hat{\nu}+D \hat{e} \times \hat{\nu}}{1+a \hat{e} \cdot \hat{\nu}} .
$$

The components of this polarization can readily be measured when the outgoing baryon $b$ is a hyperon which undergoes a subsequent weak decay $b \rightarrow b^{\prime} \pi$ with a non-zero decay 
asymmetry parameter $\alpha_{b^{\prime}}$. The distribution of the $b^{\prime}$ direction relative to any axis defined by a unit vector $\hat{i}$ is given by

$$
\frac{1}{\Gamma} \frac{d \Gamma}{d \Omega_{b^{\prime}}}=\frac{1}{4 \pi}\left(1+\mathrm{S}_{\mathrm{i}} \alpha_{b^{\prime}} \hat{i} \cdot \hat{b}^{\prime}\right)
$$

where $\mathbf{S}_{\mathbf{i}}=\left\langle\mathbf{P}_{\mathbf{b}} \cdot \hat{i}\right\rangle$ is the average polarization of $b$ in the $\hat{i}$ direction. Conceptually, it is advantageous to employ the orthonormal basis

$$
\begin{aligned}
& \hat{\alpha}=\frac{\hat{e}+\hat{\nu}}{\sqrt{2(1+\hat{e} \cdot \hat{\nu})}}, \\
& \hat{\beta}=\frac{\hat{e}-\hat{\nu}}{\sqrt{2(1-\hat{e} \cdot \hat{\nu})}}, \\
& \hat{\gamma}=\hat{\alpha} \times \hat{\beta}
\end{aligned}
$$

Experimentally, it may be more advantageous to determine the polarization components along one or more of the outgoing particle directions $(\hat{e}, \hat{\nu}, \hat{b})$.

To gauge the importance of the recoil contributions, in Fig 1 we compare values of several integrated observables calculated from our expressions with the corresponding zerorecoil values for the decay $\Xi^{0} \rightarrow \Sigma^{+} e^{-} \bar{\nu}$. For these calculations, we assumed $V_{u s}=0.2205$, $f_{1}(0)=1.0, f_{2}=2.6$, and $g_{2}=0.0$. Comparing values of integrated observables obtained from our expressions with exact values from tables in Ref. [5], we find that the decay rates agree to better than $1 \%$, and that polarizations and asymmetries agree to better than 0.004, the differences being almost entirely due to terms of order $\delta^{3}$. We have not included electromagnetic corrections, which are discussed in Ref. [5].

Finally, we present the analytic expressions for the integrated final state polarization to order $\delta$ in the final state rest frame, assuming real form factors. The order $\delta^{2}$ expressions can be obtained by adding the $\mathcal{O}\left(\delta^{2}\right)$ terms given in [11].

$$
\begin{aligned}
R= & R_{0}\left[\left(1-\frac{3}{2} \delta\right) f_{1}^{2}+\left(3-\frac{9}{2} \delta\right) g_{1}^{2}-(4 \delta) g_{1} g_{2}\right]+R\left(\delta^{2}\right), \\
R S_{\mathrm{e}}= & R_{0}\left[\left(2-\frac{10}{3} \delta\right) g_{1}^{2}+\left(2-\frac{7}{3} \delta\right) f_{1} g_{1}-\left(\frac{1}{3} \delta\right) f_{1}^{2}\right. \\
& \left.-\left(\frac{2}{3} \delta\right) f_{1} f_{2}+\left(\frac{2}{3} \delta\right) f_{2} g_{1}-\left(\frac{2}{3} \delta\right) f_{1} g_{2}-\left(\frac{10}{3} \delta\right) g_{1} g_{2}\right]+R \mathrm{~S}_{\mathrm{e}}\left(\delta^{2}\right),
\end{aligned}
$$




$$
\begin{aligned}
R \mathrm{~S}_{\nu}= & R_{0}\left[\left(-2+\frac{10}{3} \delta\right) g_{1}^{2}+\left(2-\frac{7}{3} \delta\right) f_{1} g_{1}+\left(\frac{1}{3} \delta\right) f_{1}^{2}\right. \\
& \left.+\left(\frac{2}{3} \delta\right) f_{1} f_{2}+\left(\frac{2}{3} \delta\right) f_{2} g_{1}-\left(\frac{2}{3} \delta\right) f_{1} g_{2}+\left(\frac{10}{3} \delta\right) g_{1} g_{2}\right]+R \mathrm{~S}_{\nu}\left(\delta^{2}\right), \\
R \mathrm{~S}_{\alpha}= & R_{0}\left[\left(\frac{8}{3}-\frac{52}{15} \delta\right) f_{1} g_{1}+\left(\frac{16}{15} \delta\right) f_{2} g_{1}-\left(\frac{16}{15} \delta\right) f_{1} g_{2}\right]+R \mathrm{~S}_{\alpha}\left(\delta^{2}\right), \\
R \mathrm{~S}_{\beta}= & R_{0}\left[\left(\frac{8}{3}-4 \delta\right) g_{1}^{2}-\left(\frac{8}{15} \delta\right) f_{1}^{2}-\left(\frac{16}{15} \delta\right) f_{1} f_{2}\right. \\
& \left.-\left(\frac{64}{15} \delta\right) g_{1} g_{2}\right]+R \mathrm{~S}_{\beta}\left(\delta^{2}\right),
\end{aligned}
$$

where

$$
R_{0}=\frac{G_{S}^{2}\left(\delta M_{B}\right)^{5}}{60 \pi^{3}}
$$

As can be seen in Ref. [2], the zero-recoil $(\delta=0)$ expression for $\mathbf{S}_{\mathbf{e}}\left(\mathrm{S}_{\nu}\right)$ is the same as that for the neutrino (electron) asymmetry for a polarized initial baryon [5]. Also, $R \mathrm{~S}_{\alpha}$ depends only on $V \times A$ cross terms, and $R S_{\beta}$ depends only on $V \times V$ and $A \times A$ terms, as required by a theorem due to Weinberg [10].

We thank J.L. Rosner for helpful comments and discussions. The continuing stimulation provided by our colleagues in the KTeV collaboration at Fermilab, especially T. Alexopoulos, A.R. Erwin, D.A. Jensen, E. Monnier, E.J. Ramberg, N. Solomey and M. Timko, is also gratefully acknowledged. This work was supported in part by the U. S. Department of Energy under grants DE-FG02-90ER40560 (Task B) and DE-FG02-95ER40896 (Task A). One of us (SB) acknowledges receipt of a U. S. Department of Education GANN Fellowship. 


\section{REFERENCES}

[1] A. Affolder et al., Phys. Rev. Lett. 82, 3751 (1999).

[2] W. Alles, Nuovo Cimento 26, 1429 (1962).

[3] P. S. Desai, Phys. Rev. 179, 1327 (1969) and references therein.

[4] V. Linke, Nuc. Phys. B12, 669 (1969).

[5] A. Garcia and P. Kielanowski, The Beta Decay of Hyperons, Lecture Notes in Physics Vol. 222 (Springer-Verlag, Berlin, 1985).

[6] H. Primakoff, Rev. Mod. Phys. 31, 802 (1959).

[7] A. Fujii and H. Primakoff, Nuovo Cimento 12, 327 (1959).

[8] J. M. Watson and R. Winston, Phys. Rev. 181, 1907 (1969). Their normalization convention differs from ours in that each spinor has the normalization $\sqrt{E+m}$ factored out into their phase space term. Also, in order to make their phase space correct to second order, one must replace $M_{b} / M_{B}$ with $\left(E_{b}+M_{b}\right) / 2 M_{B}$.

[9] We employ the metric and $\gamma$ matrix conventions of Ref. [5], so that $g_{1} / f_{1}$ is positive for neutron beta decay. They differ from those of J. D. Bjorken and S. D. Drell,Relativistic Quantum Mechanics, (McGraw-Hill, New York, 1964) only in that $\gamma_{5}$ has the opposite sign, and that there is no $i$ in the definition of $\sigma_{\alpha \beta}$.

[10] S. Weinberg, Phys. Rev. 115, 481 (1959).

[11] The correct order $\delta^{2}$ expressions are obtained by adding

$$
\begin{aligned}
R\left(\delta^{2}\right)= & R_{0} \delta^{2}\left(\frac{6}{7} f_{1}^{2}+\frac{12}{7} g_{1}^{2}+6 g_{1} g_{2}\right. \\
& \left.+\frac{6}{7} f_{1} f_{2}+\frac{4}{7} f_{2}^{2}+\frac{12}{7} g_{2}^{2}\right), \\
R \mathrm{~S}_{\mathrm{e}}\left(\delta^{2}\right)= & R_{0} \delta^{2}\left(\frac{55}{42} g_{1}^{2}+\frac{17}{21} f_{1} g_{1}+\frac{19}{42} f_{1}^{2}+\frac{4}{3} f_{1} f_{2}-\frac{10}{21} f_{2} g_{1}\right. \\
& \left.+\frac{10}{21} f_{1} g_{2}+\frac{116}{21} g_{1} g_{2}+\frac{4}{21} f_{2}^{2}+\frac{4}{3} g_{2}^{2}-\frac{16}{21} f_{2} g_{2}\right),
\end{aligned}
$$




$$
\begin{aligned}
R \mathrm{~S}_{\nu}\left(\delta^{2}\right)= & R_{0} \delta^{2}\left(-\frac{55}{42} g_{1}^{2}+\frac{17}{21} f_{1} g_{1}-\frac{19}{42} f_{1}^{2}-\frac{4}{3} f_{1} f_{2}-\frac{10}{21} f_{2} g_{1}\right. \\
& \left.+\frac{10}{21} f_{1} g_{2}-\frac{116}{21} g_{1} g_{2}-\frac{4}{21} f_{2}^{2}-\frac{4}{3} g_{2}^{2}-\frac{16}{21} f_{2} g_{2}\right) \\
R \mathrm{~S}_{\alpha}\left(\delta^{2}\right)= & R_{0} \delta^{2}\left(\frac{316}{245} f_{1} g_{1}-\frac{752}{735} f_{2} g_{1}+\frac{752}{735} f_{1} g_{2}-\frac{128}{105} f_{2} g_{2}\right) \\
R \mathrm{~S}_{\beta}\left(\delta^{2}\right)= & R_{0} \delta^{2}\left(\frac{422}{735} f_{1}^{2}+\frac{88}{49} f_{1} f_{2}+\frac{8}{35} f_{2}^{2}\right. \\
& \left.+\frac{362}{245} g_{1}^{2}+\frac{1576}{245} g_{1} g_{2}+\frac{8}{5} g_{2}^{2}\right)
\end{aligned}
$$

to $R, R \mathrm{~S}_{\mathrm{e}}, \mathrm{RS}_{\nu}, \mathrm{RS}_{\alpha}$ and $R \mathrm{~S}_{\beta}$, respectively in equation 12 . 


\section{FIGURES}
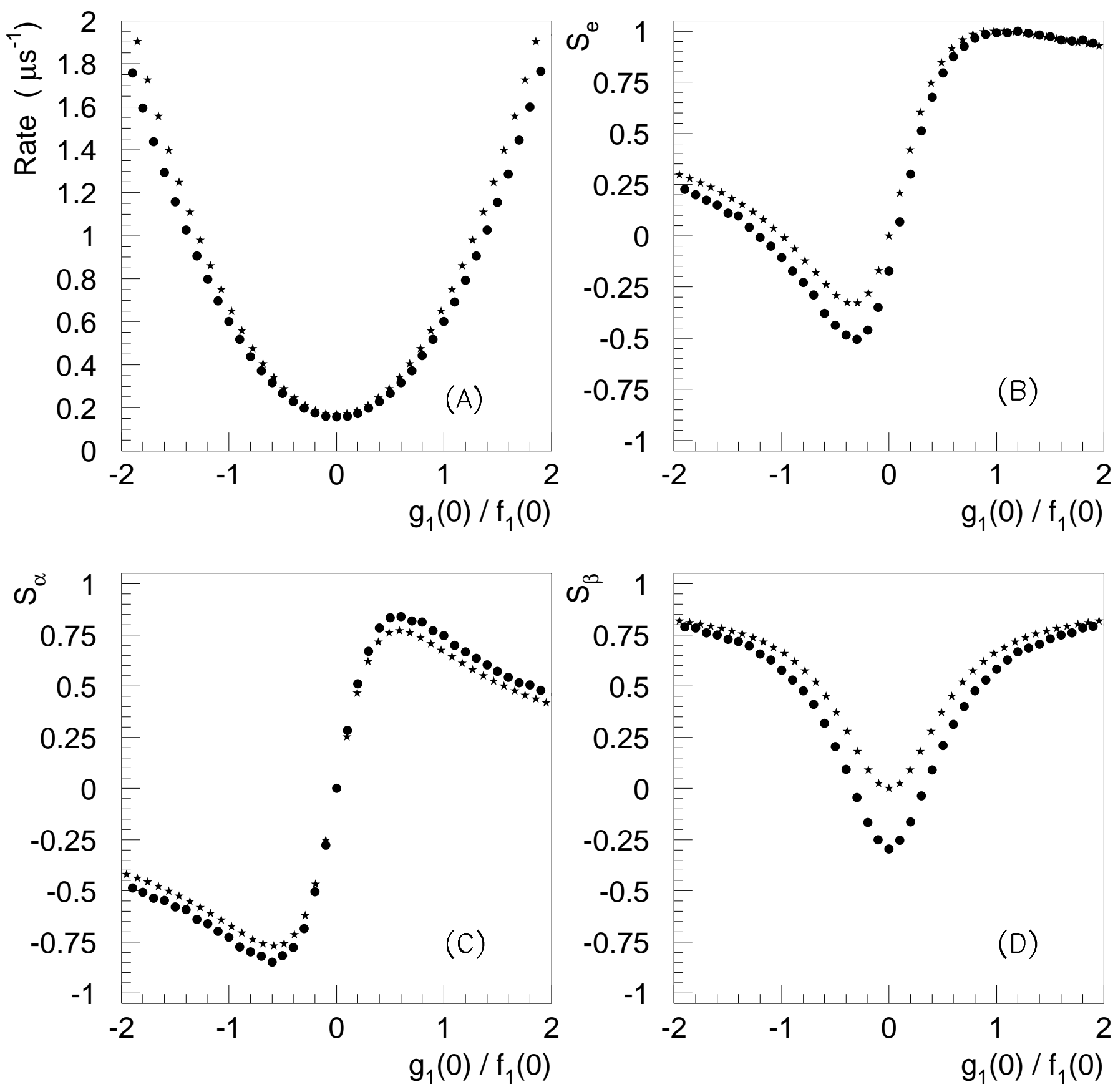
FIG. 1. Integrated observable quantities for the decay $\Xi^{0} \rightarrow \Sigma^{+} e^{-} \bar{\nu}$ as a function of $g_{1} / f_{1}$ :
A) The total decay rate $\left(\mu s^{-1}\right)$;
B) The polarization of the $\Sigma^{+}$in the $e^{-}$direction $\left(\mathrm{S}_{\mathrm{e}}=\left\langle\mathbf{P}_{\mathbf{b}} \cdot \hat{e}\right\rangle\right)$;
C) The polarization of the $\Sigma^{+}$in the $\alpha$ direction $\left(\mathrm{S}_{\alpha}=\left\langle\mathbf{P}_{\mathbf{b}} \cdot \hat{\alpha}\right\rangle\right)$;
D) The polarization of the $\Sigma^{+}$in the $\beta$ direction $\left(\mathrm{S}_{\beta}=\left\langle\mathbf{P}_{\mathbf{b}} \cdot \hat{\beta}\right\rangle\right)$.

The stars $(\star)$ are zero recoil values, and circles $(\bullet)$ are values obtained by numerical integration of our formulae ( correct to order $\delta^{2}$ ). 